\title{
CXCL5/CXCR2 axis promotes bladder cancer cell migration and invasion by activating PI3K/AKT-induced upregulation of MMP2/MMP9
}

\author{
YE GAO*, ZHENFENG GUAN*, JIAQI CHEN, HONGJUN XIE, \\ ZHAO YANG, JINHAI FAN, XINYANG WANG and LEI LI
}

Department of Urology, The First Affiliated Hospital of Medical College of Xi'an Jiaotong University, Xi'an, P.R. China

Received February 11, 2015; Accepted May 15, 2015

DOI: 10.3892/ijo.2015.3041

\begin{abstract}
Bladder cancer (BCa) is the most common malignant disease of the urinary tract system, yet the etiology is still poorly understood. Clinically, the majority of BCa patients progress to invasive disease at the final stage, leading to death. Previous investigations have demonstrated that matrix metalloproteinases (MMPs) play irreplaceable roles in tumor cell extravasation and implantation. In addition, increasing numbers of reports provide evidence that MMPs, especially MMP2 and MMP9 are monitored by various signal transduction pathways targeting tumor metastasis. Seed-and-soil theory has called to attention the importance of the tumor microenvironment in disease progression. To that end, we previously reported the key role of hypoxia in $\mathrm{BCa}$ progression. Herein, we report the role of chemokines, specifically CXCL5, is involved in $\mathrm{BCa}$ development. Though it has been reported that CXCL5 promotes $\mathrm{BCa}$ metastasis and progression, the exact mechanisms are still unknown, necessitating the need for further investigation into the role of CXCL5 in BCa. In this study, IHC staining of BCa tumor sections showed elevated expression of CXCL5 in BCa, which correlated with disease stage. Our mechanistic studies show that CXCL5 contributes to BCa migration and invasion by binding to its receptor, CXCR2, leading to the upregulation of MMP2/ MMP9 by activating PI3K/AKT signaling. This study offers vital evidence of how CXCL5 promotes BCa metastasis, and thus may potentially be used as a therapeutic target against $\mathrm{BCa}$.
\end{abstract}

\section{Introduction}

Bladder cancer (BCa) is one of the most common malignant diseases around the world with the highest recurrence rate of

Correspondence to: Professor Lei Li, Department of Urology, The First Affiliated Hospital of Xi'an Jiaotong University, 277 Yanta West Road, Xi'an 710061, P.R. China

E-mail: lilydr@163.com

${ }^{*}$ Contributed equally

Key words: bladder cancer, CXCL5, CXCR2, PI3K/AKT, migration, invasion, MMP2, MMP9 any malignancy (1). Lack of understanding of mechanisms that govern $\mathrm{BCa}$ tumor metastasis and recurrence is a major reason for its high mortality rates (2). Matrix metalloproteinases (MMPs) play irreplaceable roles in $\mathrm{BCa}$ migration and invasion $(3,4)$, which are mediated by many signal pathways including MAPK/P38 signaling (5,6), PI3K/AKT signaling (7-9), Wnt/Frizzled signaling (10) accompanied by epithelial to mesenchymal transition $(\mathrm{EMT})$, drug resistance $(11,12)$ and tumor progression. Therefore, development of BCa therapeutics may lie in understanding the roles that these signaling pathways play in disease progression.

CXCL5, also named epithelial-neutrophil activating peptide-78 (ENA-78), is a small (8-14 $\mathrm{kDa}$ ) protein, belonging to ' $\mathrm{ELR}^{+}$' subgroup of chemokines (13-15), CXCL5 is an inflammatory and angiogenic chemokine that binds to its cell-surface receptor CXCR2 to execute its roles in angiogenesis, immune response and tumor progression. Numerous reports have indicated that CXCL5 plays vital roles in cancer progression (16), including intrahepatic cholangiocarcinoma (17), prostate cancer $(18,19)$, gastric cancer $(20)$, and head and neck squamous cell carcinoma (21). Though inhibition of CXCL5 in BCa cell line T24 results in decreased migration and cell proliferation (22), it is unknown whether CXCR2, and its associated mechanisms, are necessary during this process.

PI3K/AKT signaling is an evolutionarily conserved pathway involved in physiological processes including embryonic development, cell mitogenesis, and inflammatory reactions (23-25). In addition to its physiological roles, the pathological aspect of PI3K/AKT signaling has attracted considerable research attention. Previous reports have indicated that activation of this signaling by a variety of growth factors, such as insulin, through receptor tyrosine kinases leads to tumor proliferation, metastasis, and resistance to chemotherapy $(24,25)$. Drugs targeting mTOR, an important downstream effector of PI3K/ AKT signaling, such as Sirolimus and Everolimus have been used in the battle against renal cell carcinoma, prostate cancer, and breast cancer (25-28).

Previous studies have reported that the class IB of PI3K is linked to the activation of GPCR signals (24), leading to tumor progression and metastasis. Moreover, CXCR2 is a GPCR receptor (29), which through its ligand, CXCL5, contributes to 
tumor proliferation and migration. Thus, we hypothesized that CXCL5/CXCR2 axis promotes BCa migration and invasion by activating the PI3K/AKT pathway-induced upregulation of matrix metalloproteinases.

In the present study, we used immunohistochemical analysis to assess the expression of CXCL5 in BCa tissues, and found that the expression of CXCL5 is elevated in positive correlation to the $\mathrm{BCa}$ grade. We also assessed CXCL5 expression in BCa cell lines, and the cell lines 5637 and UM-UC-2, were found to have the highest and the lowest expression of CXCL5, respectively. These cell lines were subsequently used to investigate the mechanism of $\mathrm{BCa}$ progression and metastasis. Our results indicate that in BCa cell lines 5637 and UM-UC-2, CXCL5 promotes cell migration and invasion via CXCR2; in addition, the CXCL5/CXCR2 axis induces the elevation of MMP2/MMP9 through activation of PI3K/AKT signaling. Thus, we conclude that in BCa cell lines 5637 and UM-UC-2, CXCL5/CXCR2 axis promotes cell migration and invasion by PI3K/AKT-induced elevation of MMP2/MMP9, and this axis may be targeted to inhibit tumor metastasis.

\section{Materials and methods}

Tissue preparation and immunohistochemical (IHC) staining. Tissue samples of $83 \mathrm{BCa}$ patients were obtained from the Department of Urology, First Affiliated Hospital of Xi'an Jiaotong University. The samples comprised 52 males and

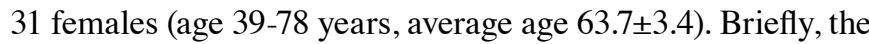
samples were fixed within $4 \%$ formalin, paraffin-embedded and $4-\mu \mathrm{m}$ thick sections used for subsequent histological analysis conducted at the Department of Pathology, First Affiliated Hospital of Xi'an Jiaotong University.

IHC was conducted using a Dako Autostainer Plus system (Dako Corp., Carpinteria, CA, USA). Tissue sections were de-paraffinized, rehydrated and subjected to 5-min pressurecooker antigen retrieval methods, 15-min endogenous enzyme block, 60-min primary antibody incubation and 30-min Dako Cytomation EnVision-HRP reagent incubation for rabbit antibodies. Signals were detected by adding substrate hydrogen peroxide using DAB (diaminobenzidine) as a chromogen followed by hematoxylin counterstaining. Negative control slices were prepared by omitting the primary antibodies. Stained (brown) cells were quantified by (number of positive cells $x 100$ / total number of cells) in 10 random microscopic (x400) fields in each slice.

Western blot analysis. Cells were harvested at $80 \%$ confluency, and washed with cold PBS three times. Total cellular protein lysates were prepared with RIPA buffer $(50 \mathrm{mM}$ Tris (pH 8.0), $150 \mathrm{mM} \mathrm{NaCl}, 0.1 \%$ SDS, $1 \%$ NP40 and $0.5 \%$ sodium deoxycholate) containing proteinase inhibitors [1\% inhibitor cocktail and $1 \mathrm{mM}$ PMSF, both from Sigma, (St. Louis, MO, USA)]. Protein $(30 \mu \mathrm{g})$ was separated on $10 \%$ SDS-PAGE gels and transferred to nitrocellulose membranes. The membranes were blocked at room temperature for $1 \mathrm{~h}$ with $5 \%$ skim milk in Tris-buffered saline ( $\mathrm{pH}$ 7.6, TBS). Polyclonal primary antibodies were applied at different dilutions (Table I) in $5 \%$ skim milk in TBS at $4^{\circ} \mathrm{C}$ overnight, followed by TBST (with Tween-20) washes. Membranes were incubated with fluorescent secondary antibodies (Licor, Rockford, IL, USA) coupled to the first antibody at room temperature in the dark for $1 \mathrm{~h}$, followed by being TBST washes, dried with neutral absorbent paper and scanned by Odyssey Detection system (Licor). MG-132 (Sigma-Aldrich, USA) was used to inhibit the proteasome-dependent degradation when necessary $(10 \mu \mathrm{M}$, $4 \mathrm{~h}$ before the protein harvest). GAPDH was used as loading control (for total cell fraction).

Real-time PCR. Cellular total RNA was isolated using TRIzol reagent (Invitrogen, Carlsbad, CA, USA) and quantitated by absorbance at $260 \mathrm{~nm}$. RNA ( $2 \mu \mathrm{g})$ was reverse transcribed using Revert Aid ${ }^{\mathrm{TM}}$ First Strand cDNA Synthesis kit (MBI Fermentas, StLeon-Rot, Germany) according to the manufacturer's protocol. For real-time PCR, we used the SYBRR Premix Ex Taq ${ }^{\mathrm{TM}}$ II system (Takara Biotechnology Co., Ltd., Dalian, China) and the Bio-Rad CFX96 ${ }^{\mathrm{TM}}$ Real-time system (Bio-Rad, CA, USA). SYBRR Premix Ex Taq II (12.5 $\mu \mathrm{l}), 1 \mu \mathrm{l}$ primer (10 $\mu \mathrm{M}$, primers, Table II), $200 \mathrm{ng}$ cDNA and $9.5 \mu \mathrm{l}$ distilled and deionized water were mixed together, followed by two stage, pre-degeneration for $95^{\circ} \mathrm{C}, 30 \mathrm{sec}$, one repeat; and PCR reaction, $95^{\circ} \mathrm{C} 5 \mathrm{sec}$ followed by $60^{\circ} \mathrm{C}, 30 \mathrm{sec}, 30$ repeats; and the third stage as dissociation, $95^{\circ} \mathrm{C}, 15 \mathrm{sec}$ followed by $60^{\circ} \mathrm{C}, 30 \mathrm{sec}$, and another $95^{\circ} \mathrm{C}, 15 \mathrm{sec}$. GAPDH was used as the loading control.

Cell culture. Human bladder cancer cell lines T24, J82, 253J, 5637, UM-UC-2, RT4, TCCSUP were obtained from ATCC (American Type Culture Collection, Manassas, VA, USA) and cultured in DMEM (for T24, J82, 253J, RT4, and UM-UC-2) or 1640 (for 5637) supplemented by $10 \%$ FBS (Invitrogen). Cells were cultured in an atmosphere with $5 \% \mathrm{CO}_{2}$ at $37^{\circ} \mathrm{C}$ (incubators: Thermo Scientific, Germany).

In order to demonstrate the roles of $\mathrm{ELR}^{+} \mathrm{CXC}$-chemokinestimulation in $\mathrm{BCa}$ cell lines, we initially used all the $\mathrm{ELR}^{+}$ CXC-chemokines (including CXCL1, CXCL2, CXCL3, CXCL5, CXCL6, CXCL7, and CXCL8, Sigma-Aldrich) to stimulate BCa cell lines for $4 \mathrm{~h}$ prior to conducting subsequent studies.

ELISA analysis. The ELISA kits for detecting CXCL5 in BCa cell line medium were obtained from Sigma (RAB0130, Sigma-Aldrich). Briefly, ELISA was performed in a blinded manner with triplicate of each sample strictly following the protocol.

Boyden chamber assay. Migration and invasion were tested by Boyden chamber assay, obtained from Millipore (Millipore, Switzerland). For migration assays, $0.2 \mathrm{ml} \mathrm{FBS-free} \mathrm{DMEM}$ medium suspension with 10,000 cells was added to the upper chamber in the 24-well plate, and $0.8 \mathrm{ml}$ FBS-free DMEM was added to the lower chamber. Following 12-h incubation, the chambers were washed with PBS ( $\mathrm{pH} 7.4)$ three times to remove the cells in the upper chamber and were fixed with $4 \%$ formalin for $15 \mathrm{~min}$, then stained with crystal violet ( $0.01 \%$ in the ethanol) for $25 \mathrm{~min}$ followed by washing three times with PBS. The cells were counted using an inverted microscope, and five visions were randomly taken in the x200 magnification, and the average number of cells analyzed. For the invasion assay, the cell suspension $(10,000$ cells/well) in the upper chamber contained $0.2 \mathrm{ml}$ mixture of FBS-free 
Table I. Antibody and reagent.

\begin{tabular}{|c|c|c|c|c|}
\hline Gene ID & Antibody & Dilutions & Species & Supplied by \\
\hline \multicolumn{5}{|l|}{ Antibody } \\
\hline NM_002994.4 & CXCL5 & $1: 300$ & Homo & Santa Cruz \\
\hline \multirow[t]{2}{*}{ NM_001014431.1 } & p-Akt & $1: 400$ & Ното & Santa Cruz \\
\hline & $\mathrm{t}-\mathrm{Akt}$ & $1: 300$ & Homo & Santa Cruz \\
\hline NM_001168298.1 & CXCR2 & $1: 300$ & Homo & Millipore \\
\hline NM_004360.3 & E-cadherin & $1: 600$ & Homo & Santa Cruz \\
\hline NM_003380.3 & Vimentin & $1: 300$ & Homo & Santa Cruz \\
\hline NM_004530.4 & MMP2 & $1: 400$ & Homo & Santa Cruz \\
\hline NM_004994.2 & MMP9 & $1: 400$ & Homo & Santa Cruz \\
\hline NM_002046.4 & GAPDH & $1: 15,000$ & Homo & ABCam \\
\hline \multicolumn{5}{|l|}{ Reagent } \\
\hline NM_001511.3 & CXCL1 & & Homo & Sigma-Aldrich \\
\hline NM_002089.3 & CXCL2 & & Mouse & Sigma-Aldrich \\
\hline NM_002090.2 & CXCL3 & & Homo & Sigma-Aldrich \\
\hline NM_002994.4 & CXCL5 & & Homo & Sigma-Aldrich \\
\hline NM_002993.3 & CXCL6 & & Homo & Sigma-Aldrich \\
\hline NM_002704.3 & CXCL7 & & Homo & Sigma-Aldrich \\
\hline NM_000584.3 & CXCL8 & & Homo & Cell Signaling Technology \\
\hline
\end{tabular}

Table II. Seqeunce of primers and siRNA.

\begin{tabular}{|c|c|c|c|c|}
\hline Gene ID & Gene & & Primers & Sapiens \\
\hline \multicolumn{5}{|l|}{ Primers } \\
\hline \multirow[t]{2}{*}{ NM_002046.4 } & $G A P D H$ & Forward: & AAC AGC GAC ACC CAT CCT C & Homo \\
\hline & & Reverse: & CAT ACC AGG AAA TGA GCT TGA CAA & Homo \\
\hline \multirow[t]{2}{*}{ NM_004360.3 } & E-cadherin & Forward: & TGC CCA GAA AAT GAA AAA GG & Homo \\
\hline & & Reverse: & GTG TAT GTG GCA ATG CGT TC & Homo \\
\hline \multirow[t]{2}{*}{ NM_003380.3 } & Vimentin & Forward: & GAG AAC TTT GCC GTT GAA GC & Homo \\
\hline & & Reverse: & GCT TCC TGT AGG TGG CAA TC & Homo \\
\hline \multirow[t]{2}{*}{ NM_004530.4 } & $M M P 2$ & Forward: & CTC ATC GCA GAT GCC TGG AA & Homo \\
\hline & & Reverse: & TTC AGG TAA TAG GCA CCC TTG AAG A & homo \\
\hline \multirow[t]{2}{*}{ NM_004994.2 } & $M M P 9$ & Forward: & TGA CAG CGA CAA GAA GTG & Homo \\
\hline & & Reverse: & CAG TGA AGC GGT ACA TAG G & Homo \\
\hline \multirow[t]{2}{*}{ NM_002994.4 } & CXCL5 & Forward: & TCA CAG AGT AGA ACC TGG GTT AGA G & Homo \\
\hline & & Reverse: & TGT GTC CCA CCA GGA CTA GAA & Homo \\
\hline \multirow[t]{2}{*}{ NM_001168298.1 } & $C X C R 2$ & Forward: & TCA CATT CCA AGC CTC ATG TCC & Homo \\
\hline & & Reverse: & GCA GAG CTC CAG CAA ATG ACA TA & Homo \\
\hline \multicolumn{5}{|l|}{ siRNA } \\
\hline \multirow[t]{2}{*}{ NM_002994.4 } & CXCL5 & Forward: & GAC GGU GGA AAC AAG GAA ATT & Homo \\
\hline & & Reverse: & UUU CCU UGU UUC CAC CGU CTT & Homo \\
\hline
\end{tabular}

DMEM/Matrigel at an 8/1 ratio (Matrigel, Sigma, USA). Cells were incubated for $36 \mathrm{~h}$ and the rest of the protocol conducted in a similar manner as the migration assay.
SiRNA and plasmid transfections. SiRNA transfections (for sequence see Table II) were used to silence the expression of CXCL5 in $5637 \mathrm{BCa}$ cell line, and pCEFL-CXCL5/Vec 
A

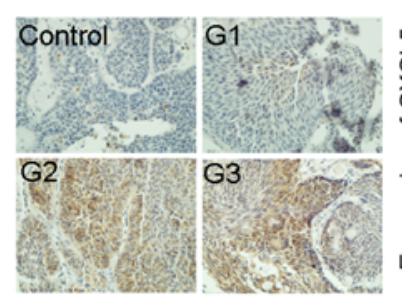

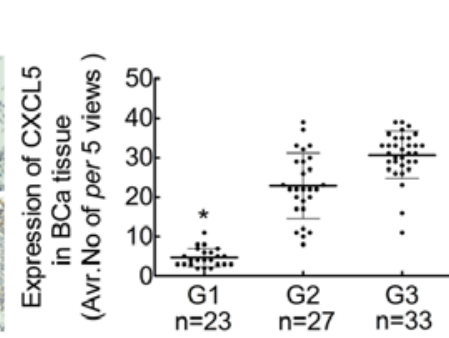

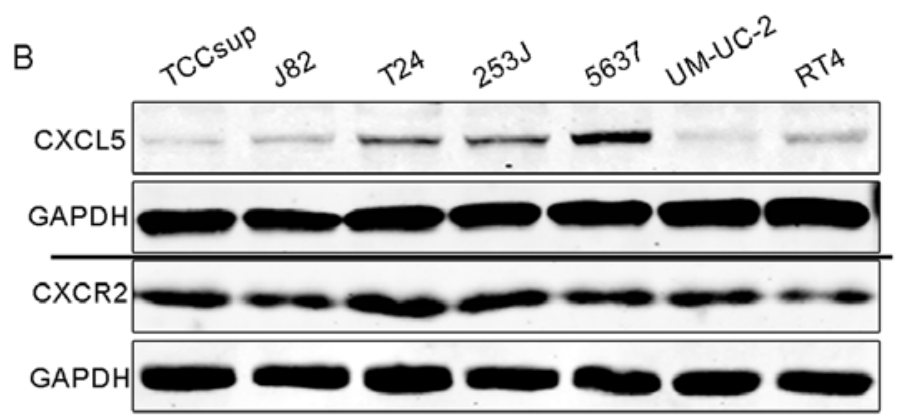

C
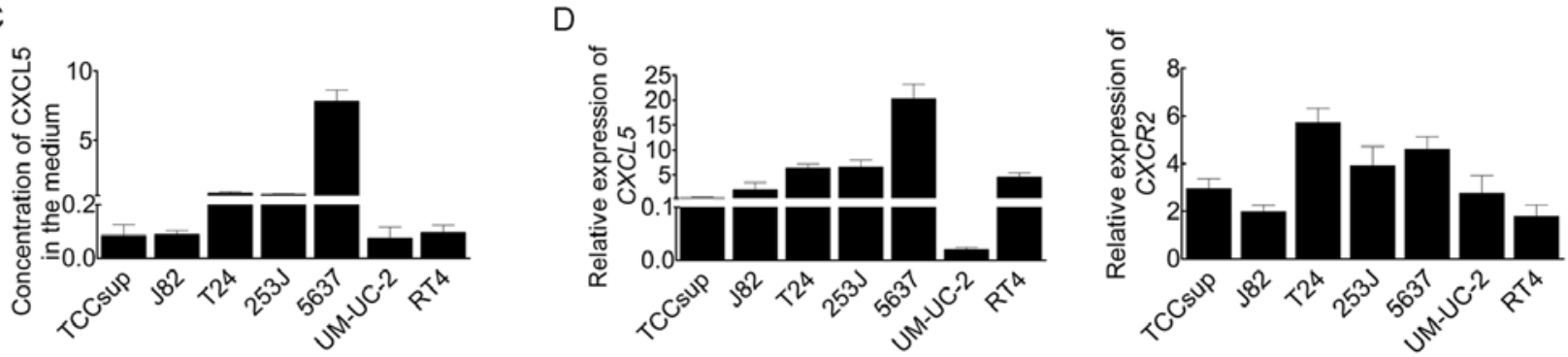

Figure 1. Expression of CXCL5 in BCa tissue and BCa cell lines. (A) IHC staining for the expression of CXCL5 in BCa tissues, manifesting as a pattern of positively correlated to BCa grade. Right, representative images of IHC staining, $\mathrm{x} 200$; left, chart of IHC staining, ${ }^{*} \mathrm{p}<0.05$. (B) Western blot analysis shows the expression of CXCL5 (top) and its receptor CXCR2 (bottom) in BCa cell lines, indicating the discrepancy expression with the highest expression of CXCL5 in 5637 and lowest expression of CXCL5 UM-UC-2. Whereas, there was no significant difference in the expression of CXCR2 in these cell lines. (C) ELISA analysis to detect the concentration of CXCL5 in the cell culture media of various BCa cell lines, manifesting as a characteristic of secreted proteins, which was consistent with the results of western blot analysis. (D) Real-time PCR analysis to monitor the expression of $C X C L 5$ and $C X C R 2$ at the mRNA level, which matched the western blot analysis data with a significant difference in CXCL5 expression but not CXCR2 in these BCa cell lines.

plasmid to overexpress CXCL5 in UM-UC-2. SiRNA for knocking down CXCR2 and Akt (both supplied by Santa Cruz Biotechnology) in 5637 and UM-UC-2 were used to further understand the roles of these two genes. Briefly, the mixture of the CXCR2/Akt1 siRNA (20 mM) and Lipofectamine 2000 (Invitrogen 1:1) were added into the cell medium for transfection over $24 \mathrm{~h}$. Fresh medium with 5\% FBS replaced the transfection medium and cells were incubated for no more than 72-h post-transfection. As a control of CXCR2 siRNA, the scramble sequence (Invitrogen) was transfected by the same method.

PT3-myr-Akt/Vec (Addgene plasmid 31789, http://www. addgene.org) was used to demonstrate the elevated phosphorylation of AKT (both T308 and S473 sites). PT3-myr-Akt/Vec and pCEFL-CXCL5/Vec were transfetced into target cell lines to generate stable clones, which had a high stable expression of p-Akt or CXCL5 compared with vector controls, respectively. Lipofectamine $^{\mathrm{TM}} 2000$ (Life Technologies, USA) was used to transfect plasmids into the target cell lines strictly accordance with the manufacturer's protocol. Western blot and real-timePCR analyses were used to monitor transfection efficiency.

Parallel to the $C X C R 2$ siRNA experiments, SB225002, the specific inhibitor of CXCR2, was used to functionally inhibit CXCR2 at a final concentration of $4 \mu \mathrm{M}$.

5-Bromo-2-deoxyuridine (BrdU) incorporation assay. 5637 and UM-UC-2 cells were plated on 24-well plates to $50-70 \%$ confluency and then stimulated with CXCL5 (10 nM) for $4 \mathrm{~h}$. BrdU was added into the medium $(3 \mu \mathrm{g} / \mathrm{ml})$. Cells were incubated for an additional $4 \mathrm{~h}$ and then rinsed $3 \mathrm{X}$ with PBS over $10 \mathrm{~min}$ to remove residual free BrdU. Cells were then fixed by
$4 \%$ paraformaldehyde for $45 \mathrm{~min}$, followed by rinsing $5 \mathrm{X}$ with PBS over $20 \mathrm{~min}$. Triton X-100 (0.1\%) was used to permeabilize the cell membrane for $15 \mathrm{~min}$ and $2 \mathrm{~N} \mathrm{HCl}$ added for 25 min to separate DNA into single strands to allow primary antibody access to the incorporated BrdU. Cells were then rinsed $3 \mathrm{X}$ with PBS over $10 \mathrm{~min}$ and non-specific epitopes blocked by $10 \%$ BSA for $20 \mathrm{~min}$. Anti-BrdU antibody (1:200) in $10 \%$ BSA was added and incubated overnight in $4^{\circ} \mathrm{C}$. Cells were rinsed $5 \mathrm{X}$ with $\mathrm{PBS}$, followed by incubation with TRTIC-labeled secondary antibody for $1 \mathrm{~h}$ in RT, and finally rinsed $3 \mathrm{X}$ with PBS to remove the free antibody. The fluorescence intensity of TRITC was monitored by SuperMicro Orifice Plate Spectrophotometer (BioTek, USA) in $547 \mathrm{~nm}$.

Statistical analysis. ANOVA test was used to analyze the statistical discrepancy in $\geq 3$ groups. Student's t-test was used to detect any statistically significant difference between 2 groups. p-values $\leq 0.05$ were considered statistically significant.

\section{Results}

CXCL5 is elevated in BCa patient tissue in correlation to tumor grade; whereas this expression pattern is not significantly correlated in BCa cell lines derived from differing grades of $B C a$. Expression of CXCL5 in BCa tissue has previously been reported (22), indicating the expression of this chemokine is elevated in positive correlation to $\mathrm{BCa}$ grade. Our IHC analysis of tumor samples derived from $83 \mathrm{BCa}$ patients confirm these previous findings (Fig. 1A). Although the discrepancy between grades II and III is not so significant, herein, we focus on the discrepancy between grade III versus grade I, and tumor tissue 
versus normal control. This increased expression of CXCL5 in BCa tissue suggested that CXCL5 likely plays a vital role in $\mathrm{BCa}$ progression.

In order to further understand the role of CXCL5 in $\mathrm{BCa}$, we assessed the expression of CXCL5 in BCa cell lines derived from different grades of $\mathrm{BCa}$ tissues using western blot and real-time PCR assays. As presented in Fig. 1B (top panel), among all the BCa cell lines, 5637 exhibited the highest CXCL5 expression, while UM-UC-2 had the lowest expression of CXCL5. These results were replicated in real-time PCR analysis (Fig. 1D, left), and ELISA analysis (Fig. 1C). Our data suggested that the expression of CXCL5 in medium grade BCa varied, but 5637 and UM-UC-2 were consistently the highest and lowest expressors, respectively. As previously reported, CXCL5 executes its functionality by binding to, and activating its receptor CXCR2. Therefore, we wanted to determine the level of expression of CXCR2 in BCa cell lines. As shown in Fig. 1B (bottom panel), we did not find a significant difference in the protein expression levels of CXCR2, although there was a difference at the mRNA level (Fig. 1D, right).

Thus far, we concluded that the expression of CXCL5 in $\mathrm{BCa}$ tissue was higher than normal tissue, and this expression was increased in positive correlation to the $\mathrm{BCa}$ grade. In addition, while the expression of CXCL5 differed in various $\mathrm{BCa}$ cell lines, the expression of CXCR 2 was not significantly different in $\mathrm{BCa}$ cells.

CXCL5 contributes to BCa cell migration and invasion. A previous study reported that a knock-down in the expression of CXCL5 in BCa T24 cell resulted in decreased cell migration (22). In addition, CXCL5 has been shown to promote tumor cell migration and invasion in breast cancer, and non-small cell lung cancer (NSCLC) (29). However, it still unknown whether increased levels of CXCL5 contribute to migration and invasion of $\mathrm{BCa}$ cells directly. To address this, we used exogenous CXCL5 to stimulate BCa for $4 \mathrm{~h}$ and assessed migration and invasion. As shown in Fig. 2A, the capacity of both migration and invasion of the two cell lines was significantly enhanced after stimulation by exogenous CXCL5. This CXCL5-stimulatory effect on migration and invasion was more enhanced in UM-UC-2 cells than in 5637 cells, which is likely due to the lower basal expression of CXCL5 in UM-UC-2 cells. In addition, due to the different basal expression of CXCL5 in 5637 cells (high expressor cells) and UM-UC-2 cells (low expressors) (Fig. 1B and D), we used siRNA transfections to silence endogenous CXCL5 in 5637 cells, and plasmid transfections to overexpress CXCL5 in UM-UC-2 cells, and to demonstrate the role of CXCL5 in tumor cell migration and invasion. Fig. 2B shows the transfection efficiency of the siRNA knock-down in 5637 or overexpression in UM-UC-2 cells. As expected, this endogenous alteration of CXCL5 significantly decreased the migration and invasion of 5637 cells (Fig. 2C, top panel) or significantly increased this effect in UM-UC-2 cells (Fig. 2C, bottom panel). Moreover, this phenomenon was more pronounced in UM-UC-2 cells.

CXCL5 promotes CXCR2-dependent BCa migration and invasion. Many investigators have shown that angiogenic chemokines, which includes $\mathrm{ELR}^{+}$chemokines, recruit endothelial cells which express the cognate receptors to these chemokines, which ultimately contributes to tumor cell proliferation and metastasis. We therefore investigated whether the increased migration and invasion we had observed thus far involved CXCR2. In order to demonstrate this point, we used siRNA transfection to silence CXCR2, or administration of SB225002, a specific CXCR2 inhibitor. As indicated in Fig. 3A, siRNA induced the decreased expression of CXCR2 at both the protein and mRNA level. Fig. 3B suggested that knocking down the expression of CXCR2 significantly attenuated the CXCL5-stimulatory effect of BCa tumor cell migration and invasion; with the basal migration and invasion also attenuated, especially in 5637 cells (top panel). As expected, SB225002 also significantly inhibited the basal (Fig. 3C) and CXCL5-stimulatory induced tumor cell migration and invasion (data not shown).

CXCL5/CXCR2 axis promotes BCa migration/invasion which involves elevation of $M M P 2 / M M P 9$. In $\mathrm{BCa}, \mathrm{MMP} 2$ and MMP9 are reported to contribute to tumor cell migration/invasion in various ways. As we had found that CXCL5/CXCR2 axis induces enhanced capacity of migration and invasion in 5637 and UM-UC-2 cells, we assessed the expression of MMP2 and MMP9 in CXCL5-stimulated 5637 and UM-UC-2 cells in the presence or absence of SB225002. We found that CXCL5-stimulated 5637 and UM-UC-2 cells manifested higher expression of MMP2 and MMP9 compared to controls (Fig. 4A). Additionally, inhibition of CXCR2 by SB225002 (Fig. 4A) or siRNA targeting CXCR2 (data not shown) significantly inhibited the CXCL5-stimulatory induced expression of MMP2 and MMP9. We also assessed the expression of E-cadherin and vimentin, key markers of EMT and known to be important in tumor cell migration and invasion, and unexpectedly there was no significant difference in the expressions of either E-cadherin or vimentin under SB225002 treatment (Fig. 4A), or CXCR2-siRNA transfection (data not shown).

CXCL5-stimulation of BCa cell-induced elevation of MMP2 and MMP9 involves activation of PI3K/AKT signaling. Elevated expression of MMPs can be monitored by various signaling pathways, including MAPK/ERK, PI3K/AKT and $\mathrm{NF}-\kappa \mathrm{B}$ pathway, among which, PI3K/AKT signaling is of interest to us. PI3K/AKT is an evolutionary-conserved pathway that plays irreplaceable and critical roles in tumor development, including tumor metastasis, proliferation, angiogenesis, and cell cycle control. In BCa, PI3K/AKT signaling has been reported to drive tumor progression. Emerging of investigation has provided evidence that MMP2 and MMP9 can be induced in many signal pathways, of which the interesting one is the PI3K/AKT signaling. As we had found that 5637 and UM-UC-2 stimulated by CXCL5 led to significantly elevated expression of MMP2 and MMP9, therefore, we hypothesized that PI3K/AKT signaling may play a role in this process. Indeed, as indicated in Fig. 4A, CXCL5 stimulation of 5637 and UM-UC-2 cells manifested higher expression of p-AKT (Th308), indicating the activation of PI3K/AKT pathway, compared with the unstimulated controls. Moreover, this elevated phosphorylation of AKT was significantly inhibited in the presence of SB225002, and accompanied by decreased expression of MMP2 and MMP9 (Fig. 4A). 

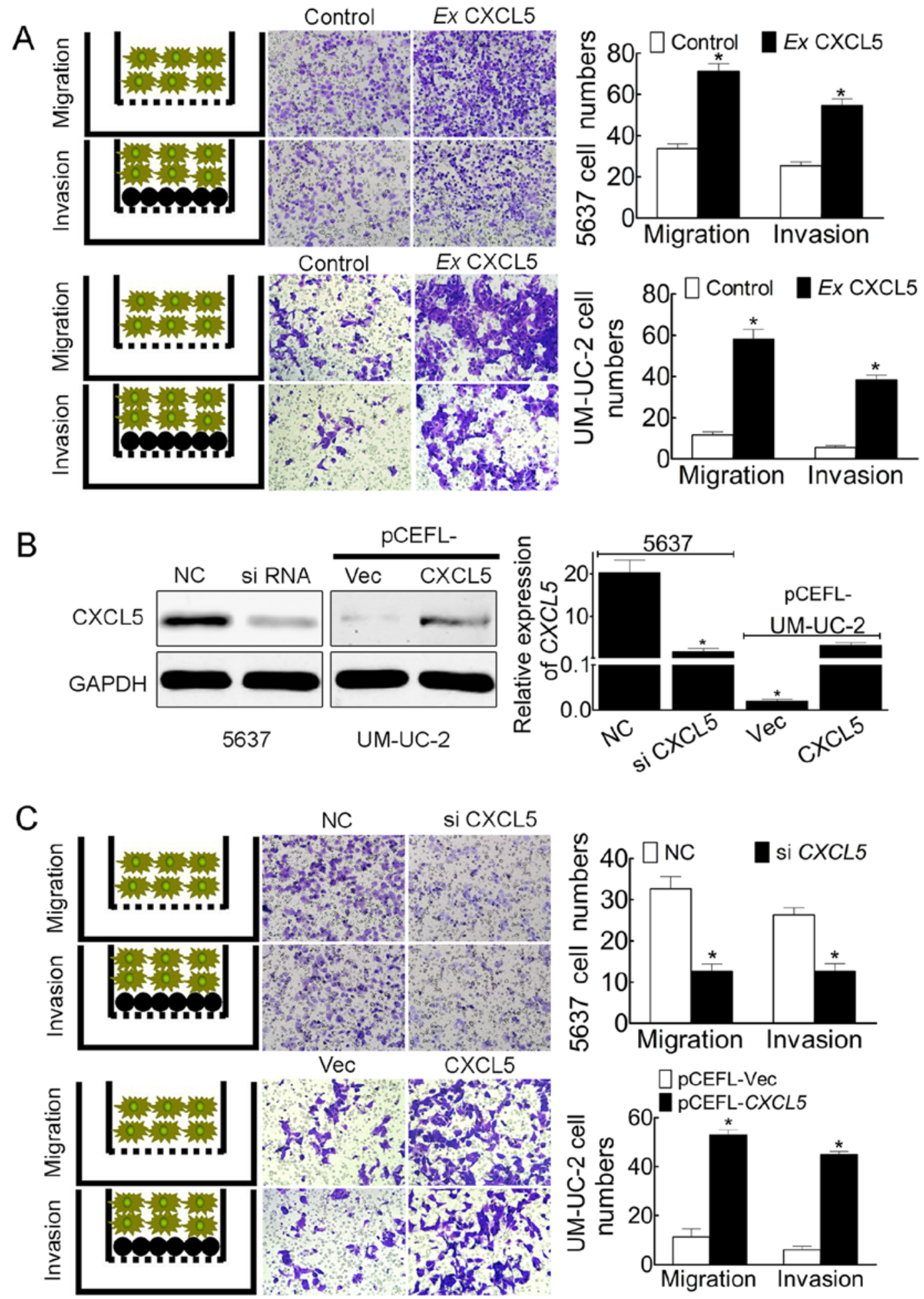

Figure 2. CXCL5 promotes BCa migration and invasion. (A) Exogenous CXCL5 (EX CXCL5) promotes 5637 and UM-UC-2 migration and invasion. Right, schematics of migration and invasion; middle, representative images of migration and invasion, $\mathrm{x} 200$; left, chart of migration and invasion, " $\mathrm{p}<0.05$. (B) Efficiency transfections to change the endogenous expression of CXCL5 in 5637 cells (high expressor) via siRNA and UM-UC-3 cells (low expressor) to overexpress CXCL5 using pCEFL-CXCL5 plasmid. Right, western blot analysis indicates the transfection efficiency in protein level; left, real-time PCR shows the transfection efficiency in mRNA level, ${ }^{*} \mathrm{p}<0.05$. (C) Endogenous CXCL5 promotes 5637 and UM-UC-2 migration and invasion. Right, schematics of migration and invasion; middle, representative images of migration and invasion, $\mathrm{x} 200$; left, chart of migration and invasion, ${ }^{*} \mathrm{p}<0.05$.

To further understand the role of AKT in CXCL5 stimulation-induced elevation of MMP2 and MMP9, we use siRNA transfections to knock down $A K T$ expression in the two cell lines, and the siRNA interference led to decreased expression of AKT at the protein and mRNA levels indicated by western blot (Fig. 4B, top) and real-time PCR analyses (Fig. 4B; bottom). The silencing of $A K T$ led to the attenuation of CXCL5 stimulation-induced expression of MMP2 
A

\begin{tabular}{lllll} 
& \multicolumn{2}{c}{5637} & \multicolumn{3}{c}{ UM-UC-2 } \\
\hline CXCR2 & si CXCR2 & NC & si CXCR2 \\
\hline GAPDH & & & \\
\hline
\end{tabular}

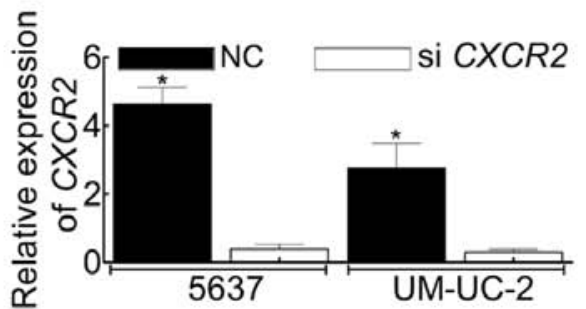

B
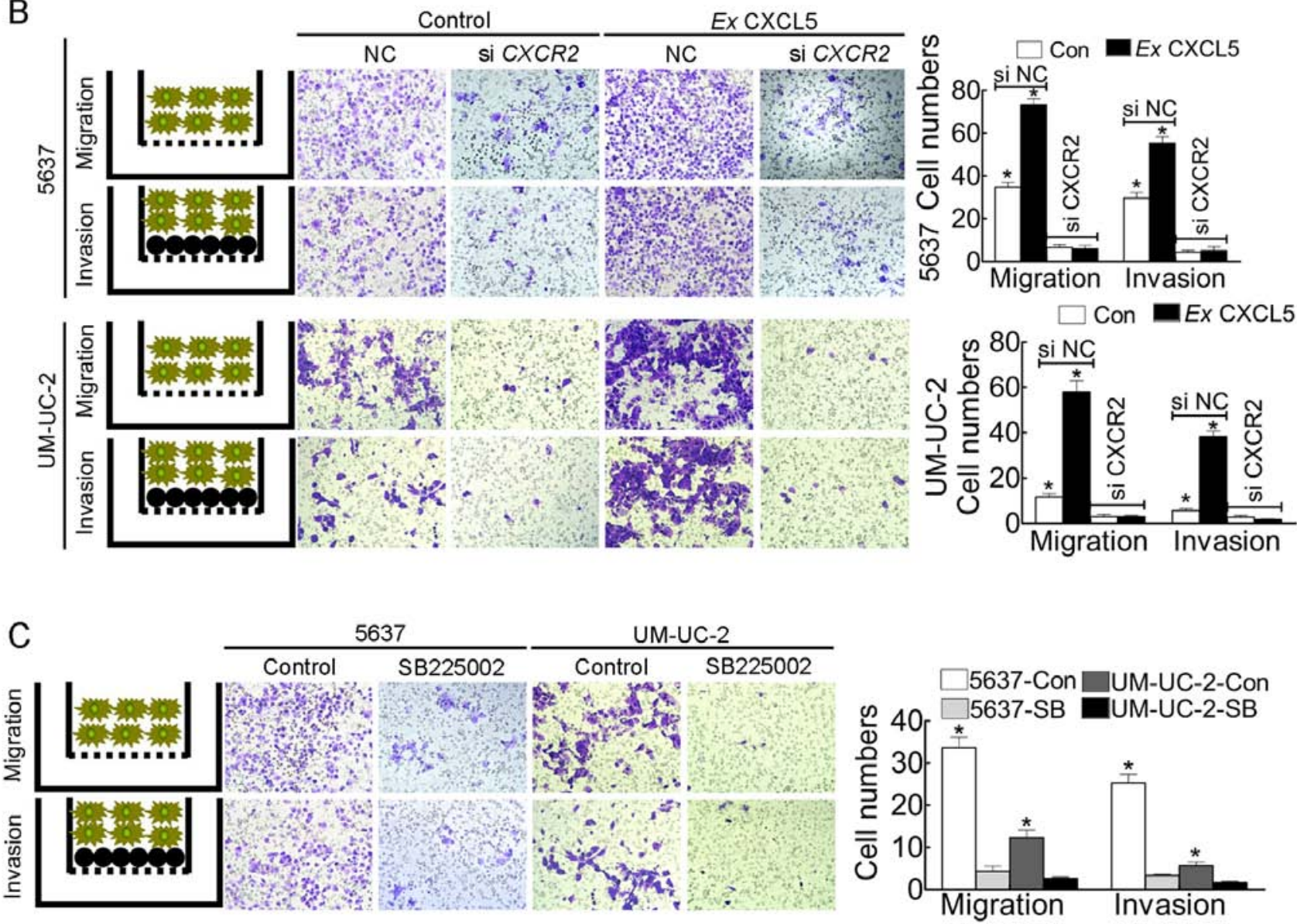

Figure 3. CXCL5 promotes CXCR2-dependent BCa migration and invasion. (A) Efficiency of silencing CXCR2 in 5637 and UM-UC-2 cells. According to Fig. 1B, expression of CXCR2 in these two cell lines was negligible, thus silencing of $C X C R 2$ was vital for investigating its roles. Right, western blot analysis shows the high efficiency of siRNA silencing; left, real-time PCR also demonstrates reduced expression, " $\mathrm{p}<0.05$. (B) Silencing CXCR2 in 5637 and UM-UC-2 cells induced attenuation of migration and invasion with, or without exogenous CXCL5. Right, schematics of migration and invasion; middle, representative images of migration and invasion, x200; left, chart of migration and invasion, " $\mathrm{p}<0.05$. (C) Functional inhibition of CXCR2 by SB225002 leads to decreased migration and invasion. Right, schematics of migration and invasion; middle, representative figures of migration and invasion, x200; left, chart of migration and invasion, ${ }^{*} \mathrm{p}<0.05$.

and MMP9 (Fig. 4C), accompanied by a decreased ability of migration and invasion of these two BCa cell lines (Fig. 4D). However, this AKT silencing by siRNA still had no effect on the expression of E-cadherin and vimentin.

Constitutive activation of PI3K/AKT signaling, which cannot be inhibited by SB225002, upregulates MMP2 and MMP9, and is accompanied by enhanced migration and invasion of parental BCa cells. One of the important sequences of activating of PI3K/AKT signaling is the recruitment of AKT to the cell membrane to bind, via its PH domain, to PIP3 following exogenous stimulations. We had seen that 5637 and UM-UC-2 stimulated by CXCL5 resulted in the elevated activation of AKT (pAKT), accompanied by elevated expression of MMP2 and MMP9, accompanied by enhanced ability of migration and invasion (Fig. 4). In order to further dissect whether the elevated expression of MMP2 and MMP9 induced by CXCL5 stimulation involved the activation of PI3K/AKT signal, we used the plasmid-tagged myristoylated sequence, which can automatically bind to cell membrane independent of the stimulating signal, leading to the activation of PI3K/AKT signaling. As noted, pAKT was very low in parental 5637 and UM-UC-2 (Fig. 4), indicating the inhibition of this signaling in parental cells. However, transfection with pT3-myr-Akt-HA significantly induced pAKT (Fig. 5A) compared to vector controls. The basic expression of MMP2 
A

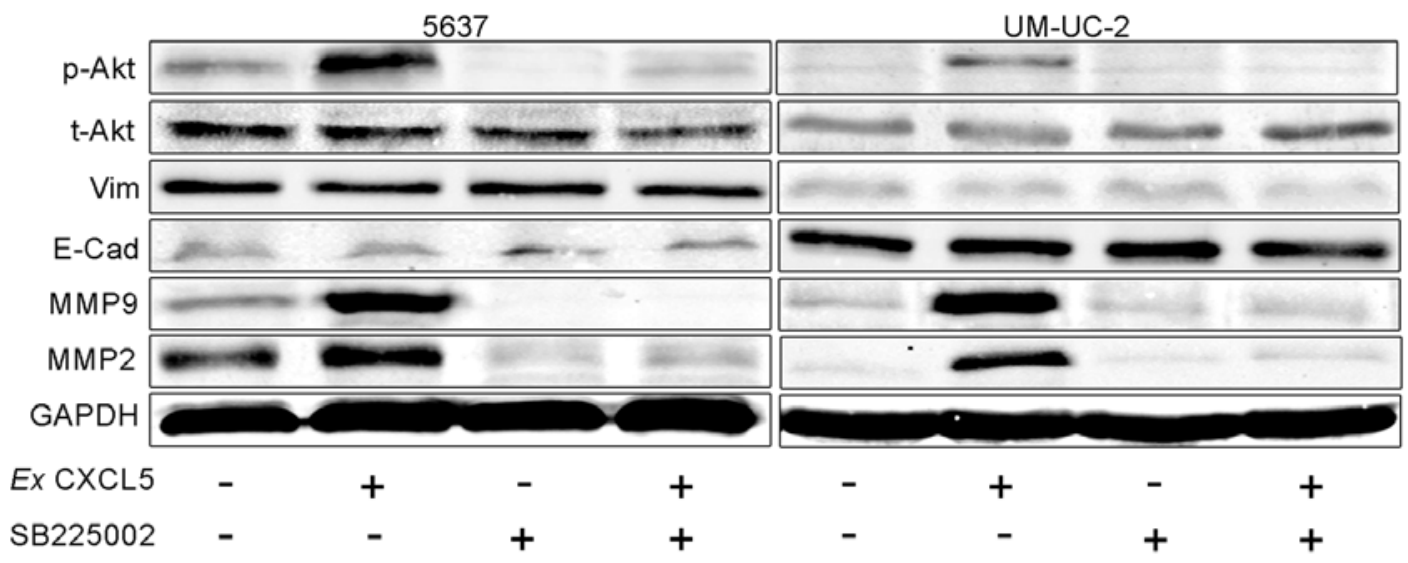

B
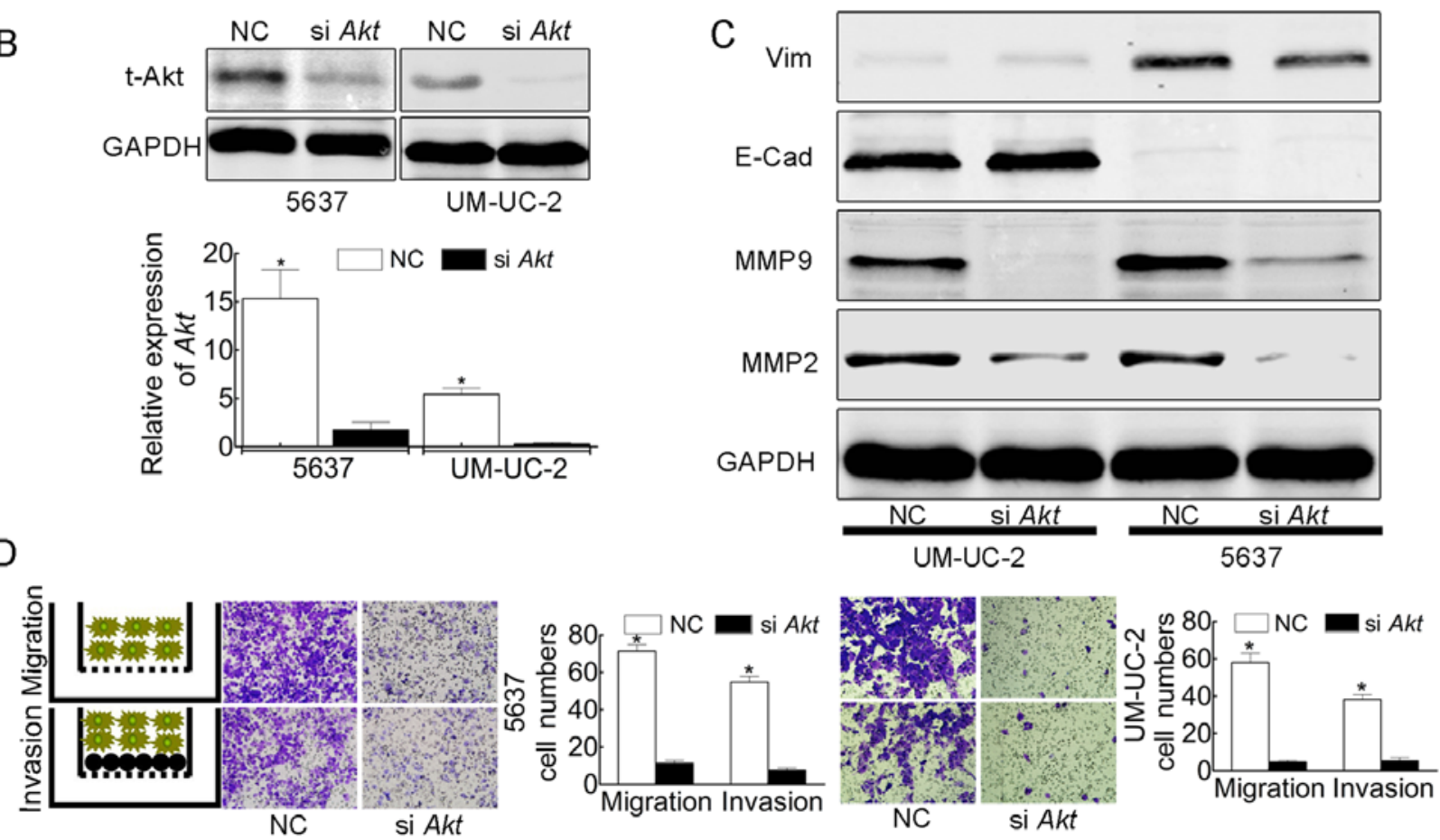

Figure 4. CXCL5 promotes BCa cell migration and invasion involves activating PI3K/AKT-induced elevation of MMP2/MMP9. (A) Western blot analysis shows that the CXCL5-stimulated 5637 and UM-UC-2 have the elevated expression of MMP2/MMP9 and pAKT. Functional inhibition of CXCR2 using SB225002 attenuates this elevation, indicating the irreplaceable role of CXCR2 in this process. However, there were no differences in expression of E-cadherin and vimentin. (B) Western blot (top) and real-time PCR (bottom) analyses show the efficiency of silencing AKT (AKT1) in the two cell lines, ${ }^{*} \mathrm{p}<0.05$. (C) Western blot analysis indicates that silencing of AKT in the CXCL5-stimulated cell lines attenuates the CXCL5-stimulating-induced elevation of MMP2/ MMP9 with no effect on E-cadherin and vimentin. (D) Boyden chamber assay shows that silencing of AKT in the two CXCL5-stimulated cell lines attenuates the CXCL5-stimulating-induced migration and invasion; right, schematics of Boyden chamber assay; middle, representative images of assay; left, chart of chamber assay, ${ }^{\mathrm{p}}<0.05$.

and MMP9 were also increased in myr-Akt-transfected cells compared to vector controls (Fig. 5B). In addition, the expression of MMP2 and MMP9 was decreased in the presence of SB225002, whereas, this inhibitor had no effect on the expression of MMP2 and MMP9 in the myr-Akt-transfected cells. This suggests that the CXCL5-stimulation-induced elevation of MMP2 and MMP9 is dependent on the activation of PI3K/AKT signaling. The functional role of this transfection is shown in Fig. 5C.

CXCL5 is the only ELR ${ }^{+}$CXC-chemokine that promotes migration and invasion of both 5637 and UM-UC-2 cells. $\mathrm{ELR}^{+} \mathrm{CXC}$-chemokines induce angiogenesis by contributing to endothelial cell recruitment, and proliferation, through interacting with CXCR2. As other $\mathrm{ELR}^{+}$chemokines can also bind and activate CXCR2, we investigated whether other $\mathrm{ELR}^{+} \mathrm{CXC}$-chemokines could induce the enhanced migration and invasion seen thus far in the two BCa cell lines. We stimulated 5637 and UM-UC-2 cells with CXCL1, CXCL2, CXCL3, CXCL6, CXCL7, and CXCL8 in a similar manner as with CXCL5. Boyden chamber assay showed that there were significant discrepancies in the migration and invasion capacity of these two cell lines upon stimulation by other $\mathrm{ELR}^{+}$chemokines. Only CXCL5 consistently enhanced migration and invasion of both 5637 and UM-UC-2 cells, as indicated in Fig. 6A. 
A

B
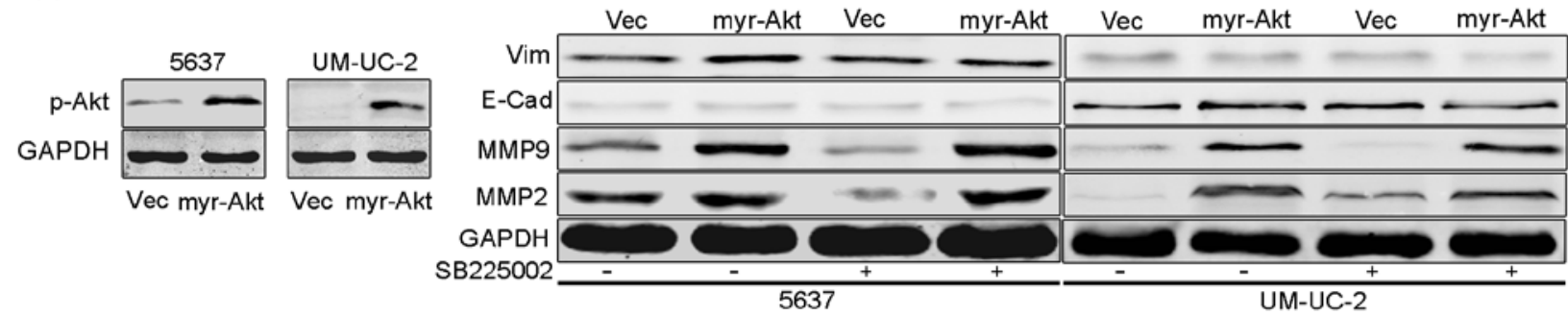

C
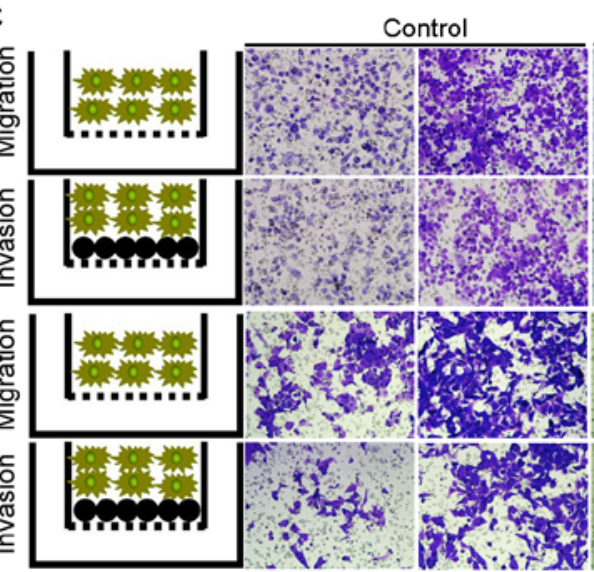

SB225002
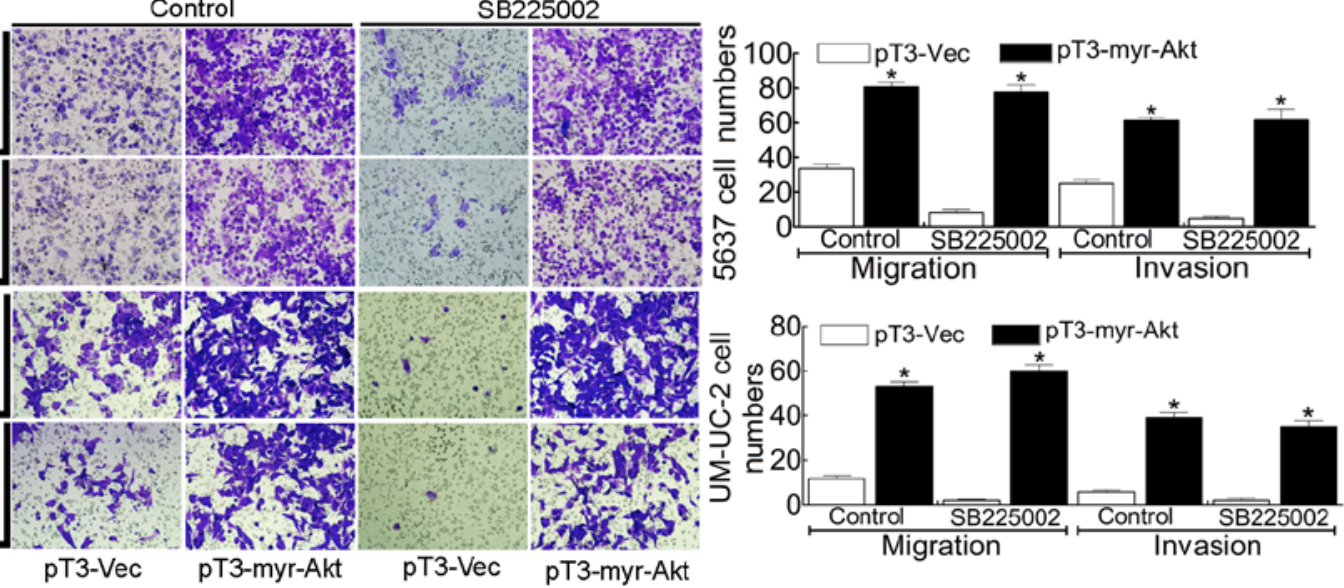

Figure 5. Activation of PI3K/AKT signaling induced enhanced ability of migration and invasion, which cannot be inhibited by SB225002. (A) Western blot analysis shows the elevated expression of pAKT in the two cell lines induced by pT3-myr-Akt transfection. (B) Western blot analysis shows that pT3-myr-Akt transfection in the two cell lines induced elevated expression of MMP2/MMP9, with no effect on E-cadherin and vimentin, which cannot be inhibited by SB225002. (C) Boyden chamber assay indicates that elevation of pAKT in the two cell lines enhances migration and invasion, which cannot be inhibited by SB225002. Right, schematics of chamber assay; middle, representative images of chamber assay; left, charts of chamber assay, ${ }^{*} \mathrm{p}<0.05$.

A
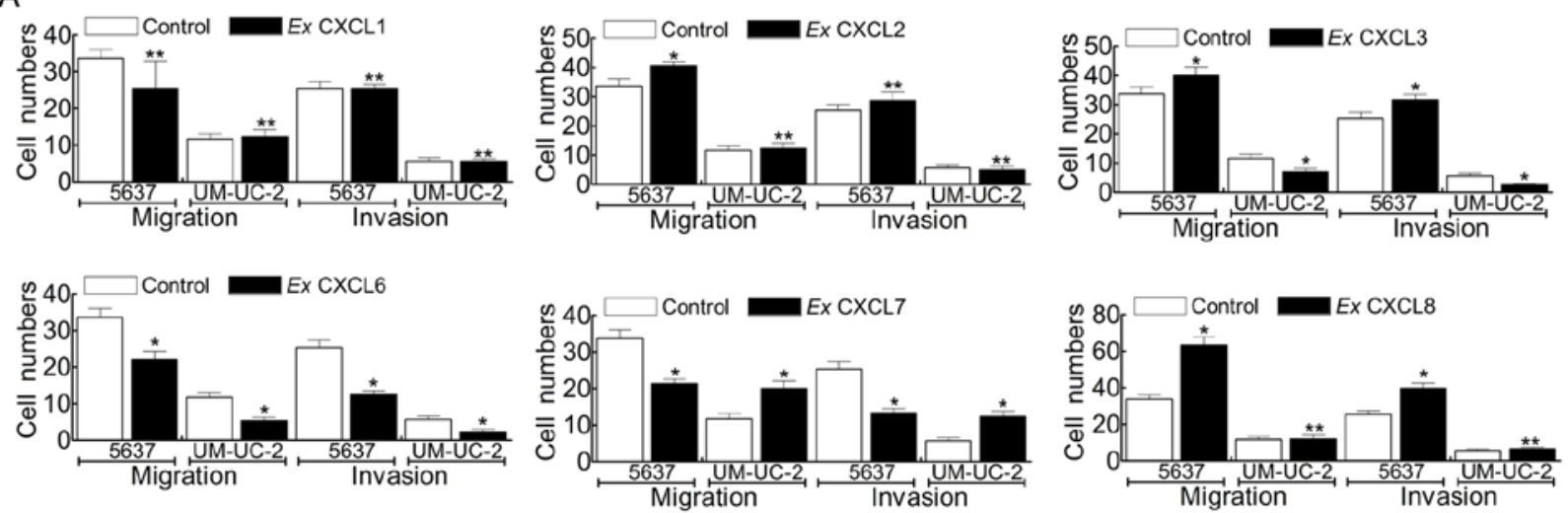

B

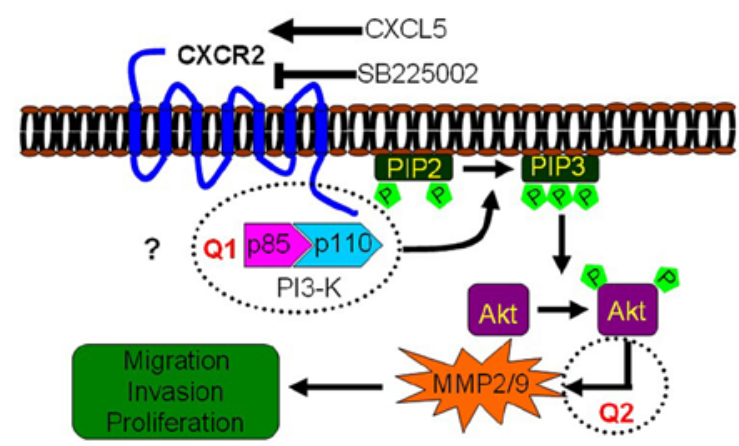

Figure 6. CXCL5 is the only ELR ${ }^{+}$CXC-chemokine that contributes to migration and invasion of both 5637 and UM-UC-2 cell lines. (A) Charts of Boyden chamber assay for migration and invasion induced by other ELR ${ }^{+}$CXC-chemokines, indicating that CXCL5 is an exception that consistently contributes to the migration and invasion of both 5637 and UM-UC-2, ${ }^{*} \mathrm{p}<0.05,{ }^{* *} \mathrm{p}>0.01$. (B) Schematic summary of the present study. In BCa cell line T24 5637 and UM-UC-2, CXCL5 binds to the membrane receptor CXCR2, leading to the activation of PI3K/AKT signaling in a currently unknown manner as indicated by 'Q1', and this CXCR2 binding can be inhibited by SB225002. Activation of PI3K/AKT signaling results in the elevation of MMP2/MMP9 via a currently unknown mechanism as indicated by Q2, leading to the enhanced ability of migration and invasion of BCa. 


\section{Discussion}

A major hindrance in the efficacy of cancer therapeutics is tumor metastases to distant organs. Extravasation into interstitial tissue space after basement membrane degradation by matrix metalloproteinases (MMPs) is one of the first, and a necessary step, for metastasis. Previous investigations have indicated that numerous signal pathways are involved in the elevated expression of MMPs. Our study presents novel findings showing that CXCL5, as a component of tumor microenvironment, contributes to the elevated expression of MMPs by binding to CXCR 2 and involves PI3K/AKT signaling, leading to tumor migration and invasion in $\mathrm{BCa}$.

Chemokines are an important component of tumor microenvironment, thus have attracted research interests in the recent years. As the second subgroup of chemokines, the role of CXC-chemokines is reported in almost all processes involved in tumor progression in many cancer types. CXC-chemokines are divided into two subgroups according to whether they express an ELR motif. Chemokines with an ELR motif (ELR ${ }^{+}$ chemokines) are angiogenic factors including CXCL1, CXCL2, CXCL3, CXCL5, CXCL6, CXCL7, and CXCL8; whereas, chemokines lacking an ELR motif (ELR chemokines) inhibit angiogenesis (13). However, CXCL12 is an exception, as it is an angiogenic ELR chemokine (13). In addition to their roles in angiogenesis, some $\mathrm{CXC}$-chemokines are reported to promote cancer progression directly (13). For instance, CXCL12 contributes to breast cancer metastasis to the lung directly by binding CXCR4/CXCR7 (30). In melanoma, CXCL12/ CXCR7 (30) axis promotes tumor cell proliferation. Previous reports have indicated the role of CXCL5 in BCa migration and proliferation (29). However, much work needs to be done to uncover the CXCL5-mediated mechanisms involved in $\mathrm{BCa}$ progression.

Our results are consistent with previous reports (29) that show the expression of CXCL5 in BCa tissue is increased according to tumor grade. However, this discrepancy in CXCL5 expression in $7 \mathrm{BCa}$ cell lines derived from differing grades was not as obvious as in tissue samples. Referencing American Type Culture Collection (http://www.atcc.org), the expression of CXCL5 in these 7 cell lines are not consistent with their tumor grade. This different pattern of CXCL5 expression in $\mathrm{BCa}$ tissue and cell lines, we postulate, may be explained as follows: in a tumor mass, expression of CXCL5 is not only by tumor cells, but also from stromal cells, and on the other hand, as indicated in Fig. 1C, in BCa cells, expression of CXCL5 is also manifested as a secreted protein. Therefore, IHC-monitored CXCL5 in BCa sections may include all the CXCL5 instead of only tumor cell-derived CXCL5, whereas, CXCL5 monitored by western blot and real-time PCR analyses only include the CXCL5 derived from tumor cells. Our data also suggest that CXCL5 promotes the migration of BCa cell lines (Fig. 2A and C), and BrdU incorporation indicates an enhanced ability of proliferation induced by CXCL5 stimulation (data not shown). Herein, we focus on CXCL5 stimulation-induced migration and invasion of $\mathrm{BCa}$. We noted that exogenous CXCL5 stimulation induced cell migration and invasion, and is more significant in UM-UC-2 cells than 5637 cells (Fig. 2A). This is attributed to the lower basal expression of CXCL5 in UM-UC-2 compared to 5637 cells (Fig. 1B).
CXCR2 is a typical G-protein coupled receptor (GPCR), and can be bound and activated by CXCL5. Functional inhibition or silencing by siRNA of CXCR2 (Fig. 3) attenuated the CXCL5-stimulatory induced ability of migration and invasion, indicating the irreplaceable roles in CXCL5-induced effects. Typically, PI3K/AKT signaling is initiated upon growth factor-induced activation of receptor tyrosine kinases (RTKs). Our data showed that CXCL5-stimulation induced the elevated phosphorylation of AKT (pAKT), indicating the activation of this pathway (Fig. 4A). This indicates that there is likely an unknown mediator linking CXCL5/CXCR2 axis and PI3K/AKT signaling, or cross-talk exists between these pathways. In addition, silencing $A K T$ by siRNA interference attenuates the CXCL5-stimulation-induced migration and invasion (Fig. 4D), accompanied by decreased expression of MMP2 and MMP9 (Fig. 4C). On the other hand, constitutively activating AKT signaling can mimic the CXCL5-stimulatoryinduced effects in the absence of exogenous CXCL5 (Fig. 5). Activation of RTKs by ligands induces the disassociation of p85 with p110, the respective regulatory and catalytic subunits of PI3K, and activation of $\mathrm{p} 110$ protein to phosphorylate PIP2 to PIP3. AKT1 is recruited to cell membranes through its PH domain to PIP3, ultimately leading to phosphorylation of AKT at T308 and S473 sites. Phosphorylation of PIP2 to PIP3 by PI3K to induce elevation of activation of AKT1 is the classical signaling pathway. Therefore, questions remain how the CXCL5/CXCR2 axis signaling induces the activation of PI3K/AKT pathway, indicated as Q1 in Fig. 6B.

Additionally, two other questions should be addressed in future studies. First, as shown in Fig. 6A, not all ELR ${ }^{+}$ CXC-chemokines have the potential to promote migration and invasion in $\mathrm{BCa}$, at least in 5637 and UM-UC-2 cells. These differing effects on 5637 and UM-UC-2 induced by these ELR chemokines may be due to specific cell type effects. Second, one of the most important downstream effectors of PI3K/AKT signaling is the NF- $\mathrm{KB}$ pathway, leading to tumor cell metastasis, proliferation, and EMT. However, in our study, markers of EMT-expression of E-cadherin and vimentin were not affected by activation of PI3K/AKT pathway. Additionally, the phenotype of cells stimulated by CXCL5 or constitutively-activated AKT1 did not manifest mesenchymal characteristics (data not shown). This suggests that the CXCL5/CXCR2 axis-induced elevated MMP2 and MMP9 by activating PI3K/AKT signaling may involve other signals different from NF- $\mathrm{BB}$ pathway, as indicated by Q2 in Fig. 6B.

In conclusion, the present study, outlined in Fig. 6B, provides evidence that CXCL5/CXCR2 axis contributes to migration and invasion of 5637 and UM-UC-2 BCa cell lines by activating PI3K/AKT signaling pathway-induced elevation of MMP2 and MMP9. Though there are still questions to be addressed, as tagged in Fig. 6B, Q1 and Q2, we provide the first direct evidence that links CXCL5/CXCR2 signaling to PI3K/AKT pathway; which will be of great interest to clinical researchers given the importance of PI3K/AKT signaling in the battle against cancer.

\section{Acknowledgements}

The present study was supported by National Natural Science Foundation of China grant (no. 81072107, 81472679). We thank 
LetPub for its linguistic assistance during the preparation of this manuscript.

\section{References}

1. Yan Y, Liang H, Li T, Li M, Li R, Qin X and Li S: The MMP-1, MMP-2, and MMP-9 gene polymorphisms and susceptibility to bladder cancer: A meta-analysis. Tumour Biol 35: 3047-3052, 2014.

2. Cancer Genome Atlas Research Network: Comprehensive molecular characterization of urothelial bladder carcinoma. Nature 507: 315-322, 2014.

3. Kanayama H: Matrix metalloproteinases and bladder cancer J Med Invest 48: 31-43, 2001.

4. Vargová V, Pytliak M and Mechírová V: Matrix metalloproteinases. EXS 103: 1-33, 2012.

5. Lai WC, Zhou M, Shankavaram U, Peng G and Wahl LM: Differential regulation of lipopolysaccharide-induced monocyte matrix metalloproteinase (MMP)-1 and MMP-9 by p38 and extracellular signal-regulated kinase $1 / 2$ mitogen-activated protein kinases. J Immunol 170: 6244-6249, 2003.

6. Westermarck J and Kähäri VM: Regulation of matrix metalloproteinase expression in tumor invasion. FASEB J 13: 781-792, 1999.

7. Chung TW, Lee $\mathrm{YC}$ and $\mathrm{Kim} \mathrm{CH}$ : Hepatitis B viral $\mathrm{HBx}$ induces matrix metalloproteinase-9 gene expression through activation of ERK and PI-3K/AKT pathways: Involvement of invasive potential. FASEB J 18: 1123-1125, 2004.

8. Cheng JC, Chou CH, Kuo ML and Hsieh CY: Radiationenhanced hepatocellular carcinoma cell invasion with MMP-9 expression through PI3K/Akt/NF-kappaB signal transduction pathway. Oncogene 25: 7009-7018, 2006.

9. Adya R, Tan BK, Punn A, Chen J and Randeva HS: Visfatin induces human endothelial VEGF and MMP-2/9 production via MAPK and PI3K/Akt signalling pathways: Novel insights into visfatin-induced angiogenesis. Cardiovasc Res 78: 356-365, 2008

10. Sen M, Reifert J, Lauterbach K, Wolf V, Rubin JS, Corr M and Carson DA: Regulation of fibronectin and metalloproteinase expression by Wnt signaling in rheumatoid arthritis synoviocytes. Arthritis Rheum 46: 2867-2877, 2002.

11. Yang JM, XuZ, Wu H,Zhu H, Wu X and Hait WN: Overexpression of extracellular matrix metalloproteinase inducer in multidrug resistant cancer cells. Mol Cancer Res 1: 420-427, 2003

12. Sethi T, Rintoul RC, Moore SM, MacKinnon AC, Salter D, Choo C, Chilvers ER, Dransfield I, Donnelly SC, Strieter R, et al: Extracellular matrix proteins protect small cell lung cancer cells against apoptosis: A mechanism for small cell lung cancer growth and drug resistance in vivo. Nat Med 5: 662-668, 1999.

13. Lee HJ, Song IC, Yun HJ, Jo DY and Kim S: CXC chemokines and chemokine receptors in gastric cancer: From basic findings towards therapeutic targeting. World J Gastroenterol 20: 1681-1693, 2014.

14. Mukaida $\mathrm{N}$ and Baba T: Chemokines in tumor development and progression. Exp Cell Res 318: 95-102, 2012.
15. Gorbachev AV and Fairchild RL: Regulation of chemokine expression in the tumor microenvironment. Crit Rev Immunol 34: 103-120, 2014

16. Kawamura M, Toiyama Y, Tanaka K, Saigusa S, Okugawa Y, Hiro J, Uchida K, Mohri Y, Inoue Y and Kusunoki M: CXCL5, a promoter of cell proliferation, migration and invasion, is a novel serum prognostic marker in patients with colorectal cancer. Eur J Cancer 48: 2244-2251, 2012.

17. Zhou SL, Dai Z, Zhou ZJ, Chen Q, Wang Z, Xiao YS, Hu ZQ, Huang XY, Yang GH, Shi YH, et al: CXCL5 contributes to tumor metastasis and recurrence of intrahepatic cholangiocarcinoma by recruiting infiltrative intratumoral neutrophils. Carcinogenesis 35: 597-605, 2014

18. Kuo PL, Chen YH, Chen TC, Shen KH and Hsu YL: CXCL5/ ENA78 increased cell migration and epithelial-to-mesenchymal transition of hormone-independent prostate cancer by early growth response-1/snail signaling pathway. J Cell Physiol 226: 1224-1231, 2011.

19. Begley LA, Kasina S, Mehra R, Adsule S, Admon AJ, Lonigro RJ, Chinnaiyan AM and Macoska JA: CXCL5 promotes prostate cancer progression. Neoplasia 10: 244-254, 2008.

20. Park JY, Park KH, Bang S, Kim MH, Lee JE, Gang J, Koh SS and Song SY: CXCL5 overexpression is associated with late stage gastric cancer. J Cancer Res Clin Oncol 133: 835-840, 2007.

21. Miyazaki H, Patel V, Wang H, Edmunds RK, Gutkind JS and Yeudall WA: Down-regulation of CXCL5 inhibits squamous carcinogenesis. Cancer Res 66: 4279-4284, 2006.

22. Zheng J, Zhu X and Zhang J: CXCL5 knockdown expression inhibits human bladder cancer T24 cells proliferation and migration. Biochem Biophys Res Commun 446: 18-24, 2014.

23. Toker A and Marmiroli S: Signaling specificity in the Akt pathway in biology and disease. Adv Biol Regul 55: 28-38, 2014.

24. Yang Z, Li Z, Feng H, Bian X, Liu Y and Liu Y: Enhanced growth inhibition by combined two pathway inhibitors on K-ras mutated non-small cell lung cancer cells. Zhonghua Zhong Liu Za Zhi 36 645-650, 2014 (In Chinese).

25. Polivka J Jr and Janku F: Molecular targets for cancer therapy in the PI3K/AKT/mTOR pathway. Pharmacol Ther 142: 164-175, 2014.

26. Houédé N and Pourquier P: Targeting the genetic alterations of the PI3K-AKT-mTOR pathway: Its potential use in the treatment of bladder cancers. Pharmacol Ther 145: 1-18, 2015.

27. Martini M, De Santis MC, Braccini L, Gulluni F and Hirsch E. PI3K/AKT signaling pathway and cancer: An updated review. Ann Med 46: 372-383, 2014.

28. Heavey S, O'Byrne KJ and Gately K: Strategies for co-targeting the PI3K/AKT/mTOR pathway in NSCLC. Cancer Treat Rev 40: 445-456, 2014.

29. Saintigny P, Massarelli E, Lin S, Ahn YH, Chen Y, Goswami S, Erez B, O'Reilly MS, Liu D, Lee JJ, et al: CXCR2 expression in tumor cells is a poor prognostic factor and promotes invasion and metastasis in lung adenocarcinoma. Cancer Res 73: 571-582, 2013.

30. Würth R, Bajetto A, Harrison JK, Barbieri F and Florio T: CXCL12 modulation of CXCR4 and CXCR7 activity in human glioblastoma stem-like cells and regulation of the tumor microenvironment. Front Cell Neurosci 8: 144, 2014. 